\title{
THE DEVELOPMENT OF THE EDINBURGH MODULAR ARM SYSTEM
}

\author{
David Gow \\ Director of Rehabilitation Engineering Services, Lothian Primary Care NHS Trust, Princess Margaret Rose \\ Orthopaedic Hospital, Fairmilehead, Edinburgh, EH10 7ED
}

\section{BACKGROUND}

Historically, the major research programme undertaken by the Bioengineering Centre in Edinburgh was the development of pneumatic powered limb prostheses for children affected by Thalidomide. From 1963 to the early 1980 's the Centre's main prosthetic work was the design, development and fitting of carbon dioxide powered limbs to children from Scotland and Northern Ireland. This work was carried out under the leadership of Professor David Simpson. Work on these prostheses was eventually discontinued and new work began on body powered and electrically powered components for unilateral amputees.

\section{INTRODUCTION}

In 1986 the prosthetic programme became the responsibility of the author who rationalised the work to combine the Centre's various strands of prosthetic research into an integrated programme, based on modularity. It was recognised that despite the undoubted merits of the gas powered arms, there were many reasons for altering the direction of the work of the Centre. Not least was the observation that there was no real, continuing demand from the users of the pneumatic prostheses. Undoubtedly a number of factors influenced this including compensation packages and other outside pressures affecting the lives of the users and their families. From a functional viewpoint, however, it was observed that the Simpson arms were complex and required a high degree of maintenance. In addition, the supply of pneumatic gas cylinders was inconvenient and cumbersome but the core reasons centred on limitations in functionality when compared to using the lower limb with the inherent tactile acuity.

Logistically the Edinburgh work had concentrated on designing for bilateral absence and this limited the general applicability of the resultant technology. There had also been significant developments in electrical prostheses which had shifted the focus of prosthetic research and service provision to this power medium. There were still, however, serious gaps in the available hardware. For example, a survey of available componentry in the mid 1980 's indicated a shortfall in the availability of components for functional partial hand replacement or for shoulder disarticulation or forequarter absence. It was also clear that the commercial systems which were available were broadly incompatible with each other and offered the Scottish Health care system a variety of components with different cosmetic appearances and with major planned obsolescence, in the form of children's prostheses and passive prostheses with work hardenable fingers.

\section{DESIGN GOALS}

In particular, consideration was given to looking at designing versatile componentry to encompass as many of the patient groups as possible. It was felt that it should be possible to develop common components for use with different power regimes without compromising function. This work lead to the concept of designing components with structural neutrality to allow the elimination of left and right handed units and the design of modules for the most 
distal level of absence. The end result was hoped to be the transferability of components between age groups with for example adult elbows being used as children's shoulders. Simply stated, the design aims can be summarised as follows:

designing based on the "shortest and smallest" principle "neutral symmetry of form to eliminate "handedness"

transferability of parts between power regimes and age ranges to reduce component count

development of a "kit of parts" approach to allow flexibility, experimentation and re-use

\section{INITIAL WORK}

Initially there was a desire to look at developing electrically powered versions of arm devices based on the Simpson-Edinburgh pneumatic arms. The work was originally promulgated at the First International Workshop of Rehabilitation Robotics in Ottawa, Canada in June 1988 [1]. Prototype units were built and research grants obtained from the Scottish Office's Chief Scientist in 1990 and work continued full time until 1995 when funding eventually ceased. It became clear from early fittings that although the work showed considerable promise there were serious limitations to the degree of modularity and the prototype was only suitable for adult males. In addition the nature of the structural design meant that there was little or no room for on-board batteries

The power of the concept of modularity was illustrated when a solution meant to alleviate size problems in partial hands was applied to the full arm system with exciting results. One of the aims of the modular approach was to try and produce powered partial hands for adults and children to meet the perceived clinical need. A number of trial fittings in Edinburgh, Örebro and Gothenburg had shown that the design was only suitable for patients aged nine or over [2]. In attempting to find a design which would allow smaller prostheses to be developed a hand was built from powered prosthetic digits. The thumb and finger phalanges were constructed from housings containing motors and gearboxes driving worm and wheel pairs. Unlike previous designs, however, the worm wheel was held fixed and the motor/gearbox phalange rotated instead giving a compact and neat prosthetic actuator system. Using this approach, prostheses suitable for children of four years of age became possible. In addition the hands were structurally neutral and left and right prostheses could be built from a "kit of parts".

When this same design idea was applied in an attempt at solving the perceived problems of arm development, larger power units were constr ucted to give elbow and shoulder joints. The motors and gearboxes could be fitted inside a carbon fibre tube thus freeing up valuable space around the diameter which could be used to mount batteries and effectively created an endoskeltal system. In addition the compact nature of the design allowed shorter actuators to be built and allowed limb segments to be cut to length to suit children and adults. The modular approach seemed to yield the same advantages for high level absence as it did for more distal absence

\section{CURRENT STATUS}

Although external funding ceased in 1995, work has continued on a part time basis as part of the organisation's service development strategy. This has meant that progress is slower than we would have preferred, and to some extent the work has concentrated on the externally powered aspects of the modular concept. To date the two major manifestations of these design principles have been the development of a powered prosthetic digit system known as PRODIGIIS ${ }^{I M}$ and the construction of an endoskeletal arm system known as the Edinburgh Modular Arm System or EMAS. A prototype for high level unilateral absence was fitted in August 1998 to a 47 year old man. The prosthesis contained powered shoulder, elbow, wrist and hand functions and was controlled by microswitches and pressure pads activated by residual movement of the acromion The prosthesis is still in working 
order over 10 months later. Redesign work and laboratory testing are currently underway based on the valuable feedback obtained from the user's comments Further volunteer patients have now been identified to test all levels of the system's configurations.

\section{FUTURE PLANS}

A small clinical trial of the partial hand configurations of the Edinburgh Arm System is planned in conjunction with colleagues in Nottingham, England Other Centres in Chicago, Örebro, Gothenburg and Belgium have expressed an interest in participating.

Further fittings of the complete arm system are planned before the year's end to an adult male in Manchester, England, an adult female in Glasgow, Scotland and to an 11 year old boy in Örebro, Sweden. New degrees of freedom for the wrist and shoulder are at the design stage

Further work on body powered and passive prostheses is underway to test the possibilities of modular transferability further:

Researchers at the Bioengineering Centre are participating in a European Union project TOMPAW in collaboration with colleagues from Sweden and England.

\section{REFERENCES}

1. Gow, D. "Physiologically Appropriate Control of a Multi-Degree of Freedom Upper Limb Prosthesis - or Where Prosthetics meets Robotics" in Proceedings of the First International Workshop on Robotic Applications in Medical and Health Care, Ottawa, Canada 1988

2. Gow, D. , Geggie, C., Douglas, W., Philipson, L. \& Peter son, S-O. "A Simple Prosthesis for Transcarpal Absence of the Hand in Children - The Reach Hand" in Proceedings of the 2nd European Conference on the Advancement of Rehabilitation Technology, Stockholm, Sweden 1993 